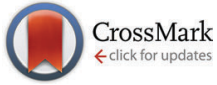

Cite this: Phys. Chem. Chem. Phys., 2016, 18, 27165

Received 26th July 2016, Accepted 30th August 2016

DOI: $10.1039 / c 6 c p 05181 b$

www.rsc.org/pccp

\section{Multivalent bonds in self-assembled bundles of ultrathin gold nanowires $\dagger$}

\author{
B. Reiser $\neq$ D. Gerstner $\neq$ L. Gonzalez-Garcia,* J. H. M. Maurer, I. Kanelidis and \\ T. Kraus*
}

\begin{abstract}
Ultrathin gold nanowires are unusual colloidal objects that assemble into bundles with line contacts between parallel wires. Each molecule in the contact line interacts with many ligand and solvent molecules. We used X-ray scattering and electron microscopy to study how these interactions control assembly.
\end{abstract}

Ultrathin gold nanowires (AuNWs) can be prepared using scalable wet-chemical protocols, which makes them interesting building blocks for functional materials. ${ }^{1}$ These wires have already been used as active components for surface-enhanced Raman scattering (SERS) substrates, ${ }^{2}$ sensors, ${ }^{3}$ and transparent electronics ${ }^{4,5}$ and are promising candidates for nanoscale interconnects. ${ }^{6}$ Oleylamine (OAm) that is tethered to the particles' surface serves as a ligand and provides good colloidal stability in organic solvents. ${ }^{1}$ The small diameters (below $2 \mathrm{~nm}$ ) and high aspect ratios (>1000) of AuNWs lend them high mechanical flexibility. ${ }^{7}$ It is possible to coil functionally coated AuNWs into nanosprings that store mechanical energy. ${ }^{8}$ Wires can bundle into hexagonal superstructures and adapt to complex shapes; ${ }^{9}$ this ability has been exploited to print them as conductive grids. ${ }^{4}$

Self-assembly is an elegant and cost-effective route to fabricate functional particle superstructures. ${ }^{9}$ Controlled self-assembly of AuNWs is a realistic way to fabricate hierarchical 3D architectures..$^{10}$ They could, for instance, serve as key components in self-assembled electronic circuits. ${ }^{4,11}$ Engineering selfassembled superstructures requires a substantiated understanding of nanoparticle interactions. However, the current understanding of the relevant interactions of AuNWs and other nanoparticles with characteristic dimensions in the range of $1-20 \mathrm{~nm}$ is limited. The sizes of the stabilizing ligands and the solvent molecules are on the same order of magnitude as the particle cores for such particles. The interactions between their surface

INM - Leibniz Institute for New Materials, Campus D2 2, 66123 Saarbrücken, Germany. E-mail: lola.gonzalez-garcia@leibniz-inm.de, tobias.kraus@leibniz-inm.de $\dagger$ Electronic supplementary information (ESI) available: Experimental details. TEM images of AuNWs synthesized in different solvents. See DOI: 10.1039/ c6cp05181b

\$ These authors contributed equally to this work. ligands can exceed the cores' interactions by far. ${ }^{12,13}$ Classical theories of colloidal particle-particle interactions, such as the Derjaguin-Landau-Verwey-Overbeek (DLVO) theory, do not satisfactorily describe the behavior of nanoparticles with diameters below $20 \mathrm{~nm}$; for instance, they do not consider ligand-ligand and ligand-solvent interactions. ${ }^{14}$ We believe that such particles are better understood in terms of intermolecular interactions with the concepts of multi- or polyvalency that are used in biochemistry and supramolecular chemistry. ${ }^{15,16}$ Ultrathin nanowires that form line contacts have been described as polymer analogues. ${ }^{17}$ In a figurative sense, the gold core of AuNWs would be the polymer backbone and the ligands the side chains that cause supramolecular interactions.

Because ligands dominate their behavior, the assembly of very small particles can be tuned via ligand type ${ }^{18}$ and ligand conformation ${ }^{19}$ as a step towards the rational design of particle self-assembly. But the choice of ligands that yield anisotropic particles in wet-chemical synthesis is very limited ${ }^{20}$ and ligand exchange is often challenging. Recent studies suggest that the solvent, which is relatively easy to exchange, can also influence nanoparticle assembly in dispersion. ${ }^{18,21}$ Solvent effects have previously been studied for isotropic particles but the result cannot be directly extrapolated to particles with different shapes. ${ }^{22}$ Here, we study highly anisotropic wires.

We investigated how ligand-solvent interactions affect the superstructure formation of AuNWs in dispersion using smallangle X-ray scattering (SAXS). We demonstrate that the solvent determines whether ordered superstructures form, their formation kinetics, and the wire-wire distance. We found that ligand excess in solution affects the packing density of the superstructures and propose a model based on weak intermolecular interactions to explain the solvent dependence of AuNW bundling. Experiments on AuNW growth in different solvents prove that ligand-solvent interactions also impact the anisotropic particle growth of AuNWs.

Ultrathin AuNWs were synthesized using a protocol adapted from Feng et $a l^{2}{ }^{2}$ that is described in the ESI. $\dagger$ The synthesis uses oleylamine $(\mathrm{OAm})$ to induce wire growth in $n$-hexane. During synthesis, OAm serves as structure directing agent; it adsorbs on 
the gold wires with its amine functionality, where it serves as a ligand and provides colloidal stability.

We analyzed as-synthesized AuNW dispersions in the liquid state by SAXS and found wires bundled into hexagonal arrangements with a lattice parameter of $9.2 \mathrm{~nm}$ (Fig. 1a). Bundles with a lattice parameter of $9.7 \mathrm{~nm}$ have previously been described by Loubat et $a .^{23}$ for $1.7 \mathrm{~nm}$ thick wires. Oleylamine has a length of approximately $2 \mathrm{~nm}$ in its fully stretched conformation; ${ }^{24,25}$ Loubat et al. concluded that four OAm molecules separated the gold wires inside a bundle. ${ }^{23}$ We found $0.5 \mathrm{~nm}$ shorter wire-wire distances that we attribute to stronger OAm interdigitation. The alkane chains of OAm can interlock like the teeth of a zipper as shown in Fig. 1c. What determines the extent of interdigitation?

Osmotic pressure exerted by solute molecules is a likely candidate. OAm is used in excess during synthesis, and free OAm may affect the wire-wire distance. We synthesized AuNWs, adjusted the OAm concentration as described in the ESI, $\dagger$ and studied the dispersions with SAXS (Fig. 1a and b). All SAXS patterns confirmed hexagonal packing of wires; the Bragg peaks

(a)

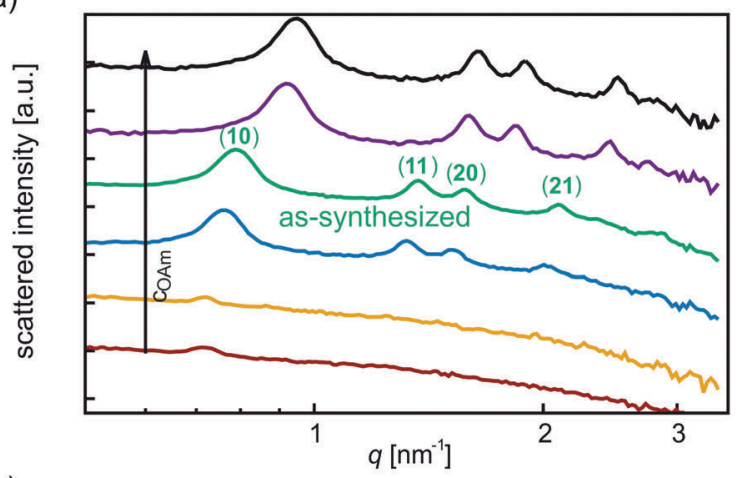

(b)

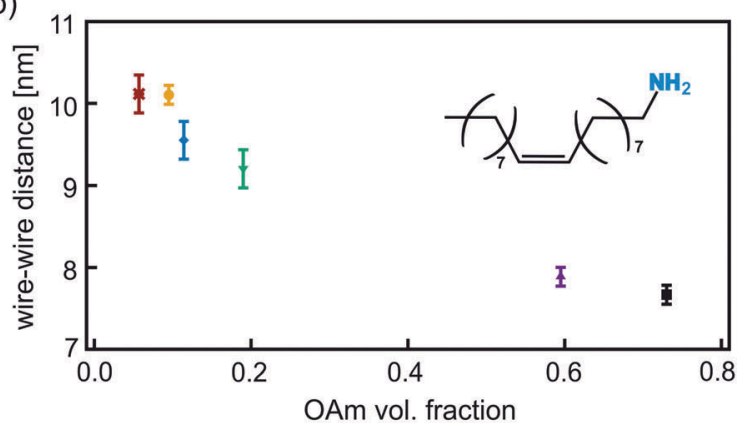

(c)
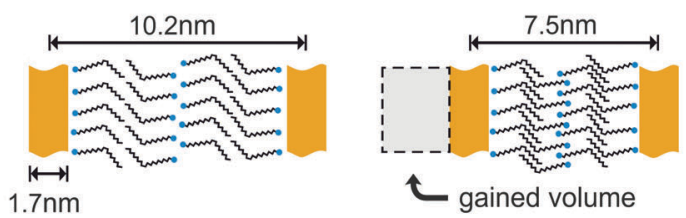

Fig. 1 (a) Normalized SAXS patterns of as-synthesized AuNWs dispersed at different volume fractions of OAm. (b) Wire-wire distance depending on free OAm volume fraction in solution. Inset: Skeletal formula of OAm. (c) Schematic illustration of OAm interdigitation for the closest and the least close wire-packing observed and of the difference in solvent volume enclosed between two wires for both cases. shifted towards larger scattering angles with increasing OAm volume fraction (Fig. 1a). The center-to-center distance of the wires was calculated from the scattering angle of the first Bragg peak (10). It decreased monotonously with the total OAm volume fraction in the dispersion (Fig. 1b). We believe that the increasing OAm concentration causes an osmotic pressure that drives out solvent molecules from inside of the bundles in order to dilute the free OAm. Interdigitation of the ligand shells thus increases with free OAm concentration as shown in Fig. 1c.

London dispersion interactions between the alkane chains of the tethered OAm molecules become stronger with increasing interdigitation. ${ }^{12,26}$ This explains the increasing intensity of the Bragg peaks with increasing OAm volume fraction (Fig. 1a): the denser the packing of the OAm between the wires, the stronger the multivalent interactions between them, and the greater the fraction of ordered bundles in the overall dispersion. Similarly variable interdigitation of alkane chains has already been observed for phospholipids in water. A phase transition from a separated to an interdigitated lipid bilayer phase is induced when increasing ethanol concentration. ${ }^{27}$

To reduce the OAm concentration while keeping the AuNW concentration constant, we washed as-synthesized AuNWs by repeated precipitation with ethanol and redispersion in the same volume of a fresh solvent. This procedure removed excess OAm and reduced the lattice parameter of the hexagonal bundles from $9.2 \mathrm{~nm}$ to $5.5 \mathrm{~nm}$ as previously reported by Moutet et al. ${ }^{28}$ The reduced spacing is consistent with a wire-wire separation of two OAm molecules as visualized in Fig. 2a. We assume that the washing procedure removed all OAm that was not directly tethered to the gold surfaces. The two remaining OAm monolayers interdigitate like a zipper in 3 dimensions. The resulting superlattice reminds of the inverted hexagonal phase of lipids in water $^{29}$ although the formation is driven not by hydrogen bonds (as for the lipids in water) but by dispersive interactions.

Bundling of AuNWs occurred slowly after redispersion in $n$-hexane (Fig. 2b). The evolution of the first SAXS Bragg peak (10) was analyzed over time (Fig. 2b inset) and its peak height, relative to the maximum peak height observed, was used as a measure for bundle formation. Peaks appeared after a few minutes; equilibrium was reached after $140 \mathrm{~min}$, when the peak heights remained constant. Other solvents led to different bundling behavior. We exchanged the solvent after washing and followed the assembly of the AuNWs in three different solvent classes: $n$-alkanes, cycloalkanes, and arenes.

SAXS patterns were recorded $24 \mathrm{~h}$ after redispersion in the new solvent to ensure equilibrium. Fig. 2c illustrates the effects of the different solvents. Gold nanowires that were redispersed in $n$-hexane and $n$-octane formed hexagonal bundles with a lattice parameter of $5.5 \mathrm{~nm}$. Cyclohexane and cyclooctane prevented ordered assembly entirely; the SAXS patterns of AuNW in these solvents were dominated by the form factor of the wires (further information can be found in the ESI $\dagger$ ). Dispersions of wires in arenes contained hexagonal superstructures; the AuNWs were packed tighter in arenes than in $n$-alkanes, with wire-wire distances of 5.2-5.3 $\mathrm{nm}$. After redispersion vigorous shaking was required to yield a stable colloidal dispersion of AuNWs 
(a)

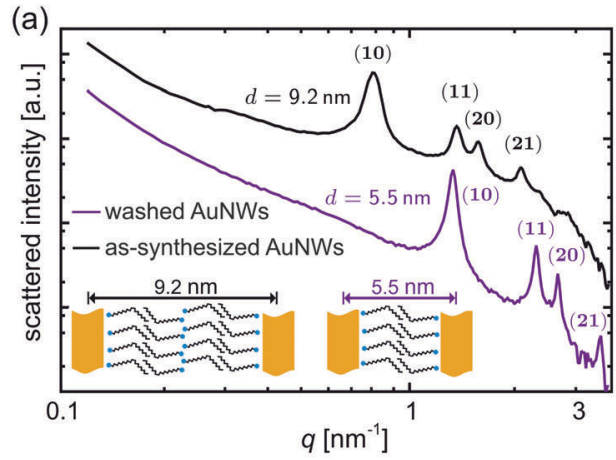

(b)

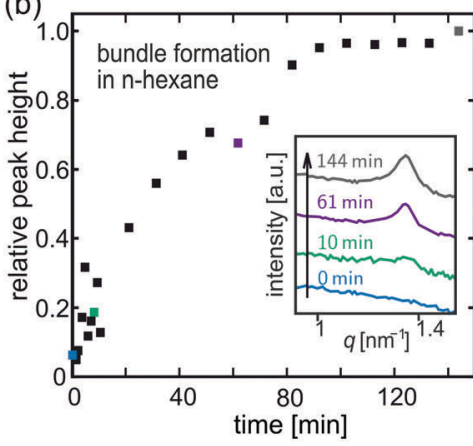

(c)

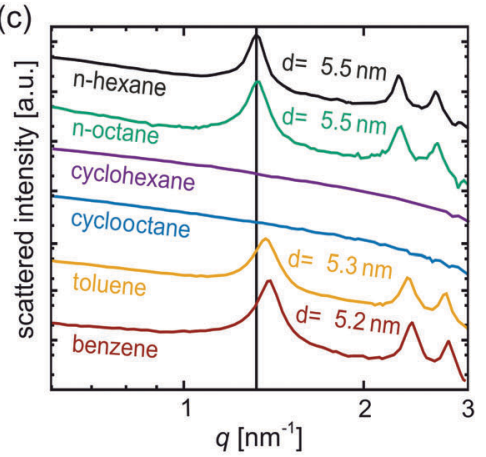

(d) Proposed model for ligand-solvent interactions

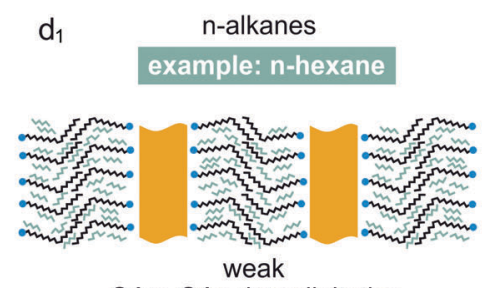

OAm-OAm interdigitation

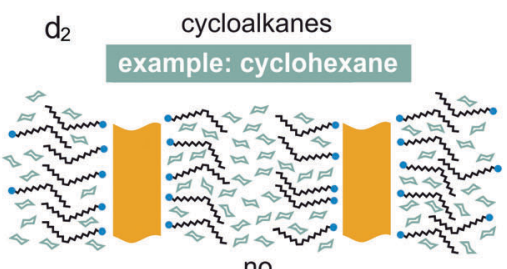

no

OAm-OAm interdigitation

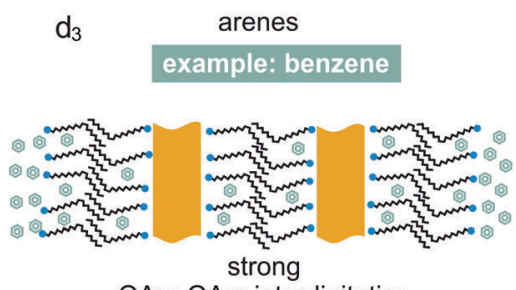

OAm-OAm interdigitation

Fig. 2 (a) SAXS patterns of as-synthesized AuNWs and washed nanowires dispersed in $n$-hexane. Inset: Schematic depiction of the ligand layer between two bundled wires. (b) Evolution of the first Bragg peak for washed AuNWs over time when redispersed in $n$-hexane. Inset: SAXS patterns at selected times. (c) SAXS patterns of washed AuNWs recorded $24 \mathrm{~h}$ after redispersion in different solvents. (d) Schematic illustration of our model for the arrangement of solvents in the OAm ligand layer of AuNWs and its influence on bundling.

in arenes, and the dispersion contained bundles immediately after shaking. Shaking probably dispersed small bundles of wires rather than dispersing individual wires that self-assembled subsequently.

The effects of solvents discussed above support our hypothesis that weak intermolecular ligand-ligand and ligand-solvent interactions dominate the AuNW-assembly behavior, similar to assembly in supramolecular chemistry. ${ }^{30,31}$ We propose a model for assembly that is based on the balance between an enthalpic term (intermolecular forces) and mixing entropy. London dispersion forces between AuNW-tethered OAm molecules are not specific; they will act between all solvent and ligand molecules. The entropic gain favors mixing of different molecules; it is the enthalpic contribution that determines whether AuNWs bundle. Solvent molecules will readily intercalate into the ligand shell if ligand-ligand and solvent-solvent interactions are not considerably stronger than ligand-solvent interactions. The intercalating solvent will influence the structure of the ligand shell. ${ }^{32,33}$ Our hypothesis is that wires bundle when the solvent efficiently intercalates neighboring ligand shell or when most solvent is expelled from touching ligand shells.

Short-chained $n$-alkanes such as $n$-hexane have sufficient conformational flexibility to intercalate an ordered OAm ligand layer and to maximize ligand-solvent interactions. ${ }^{18}$ Two wires with ordered ligand layers bundle to close the "OAm-teeth" of the "zipper" (Fig. 2d $\mathrm{d}_{1}$ ).

Cycloalkanes such as cyclohexane have less conformational flexibility. The energetically favorable "chair"-conformation of cyclohexane cannot intercalate into OAm ligand layers without disturbing their order (Fig. $2 \mathrm{~d}_{2}$ ). Disordered OAm layers interact less strongly with the OAm layers of other wires, and bundling becomes less likely.

Arenes have little conformational flexibility, but we did observe bundles in benzene and toluene. We believe that the relatively strong $\pi-\pi$-interactions between the solvent molecules ${ }^{34}$ reduced the intercalations of solvents into the OAm ligand shell. This explains both the difficulty encountered when dispersing wires in arenes and the closer packing of the wire bundles compared to $n$-alkanes. Little arene molecules remain in the tightly packed OAm bilayer (Fig. $2 \mathrm{~d}_{3}$ ).

Our interpretation is inspired by earlier models that highlight the role of the solvent's molecular shape and the solvent-solute interactions in supramolecular assembly. ${ }^{35}$

The solvent effects are so strong that we suspected them to affect the formation of AuNW during synthesis, too. Although the exact mechanism of AuNW growth is still under discussion, it is accepted that OAm plays a crucial role in the AuNWs' anisotropic growth. ${ }^{23,36}$ It is also known that solvents affect the polydispersity of spherical gold nanoparticles synthesized using OAm. ${ }^{32}$ To test whether ligand-solvent interactions affect AuNW growth, we used the solvents introduced above for wire synthesis while leaving all other parameters unchanged. Fig. 3 shows representative TEM images of the resulting wires (more TEM images can be found in the ESI, $\dagger$ Fig. S2-S7). N-Alkanes, most commonly used for AuNW synthesis, yielded large fractions of wires and some small spheres. Cycloalkanes and arenes led to much higher contents of spheres and irregularly shaped wires. Less suitable solvents apparently impair wire growth, but they do not entirely prevent it. This is compatible with the hypothesis of a soft template such as OAm micelles ${ }^{37}$ guiding anisotropic growth. 

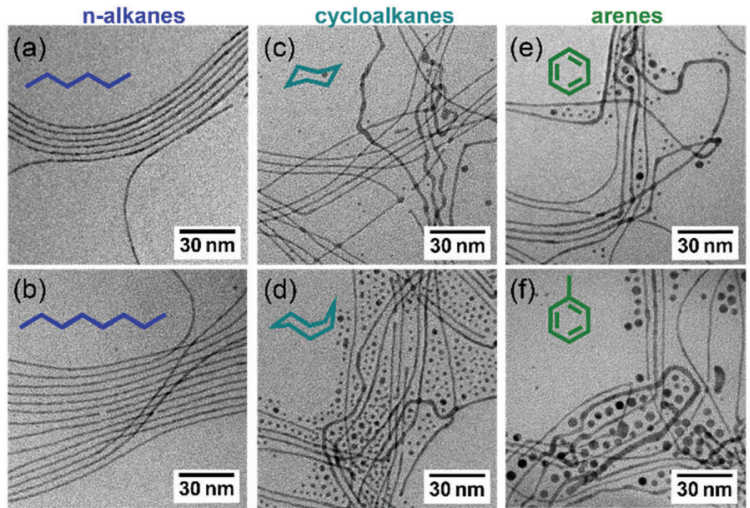

Fig. 3 TEM images of as-synthesized AuNWs in (a) $n$-hexane, (b) $n$-octane, (c) cyclohexane, (d) cyclooctane, (e) benzene and (f) toluene. Skeletal formulas of the respective solvents are given as insets.

The interactions between the soft templates may be affected by the solvent in a way similar to the wire-wire interactions discussed above. Anisotropic micelles, their interactions, and their assembly are well-understood in water, ${ }^{29}$ but little is known about the prerequisites for their formation and their dynamics in unpolar solvents. Standard techniques for the analysis of aqueous micelles like SAXS are not suitable for OAm in hexane and similar systems. Neutron scattering studies with and without metal cores could provide further insight into the dynamics of those templates.

In summary, we demonstrated that the assembly of AuNWs is dominated by intermolecular forces and strongly affected by solvents. Although the individual forces are weak, the synergy of bonds along the contact lines between wires increases their collective strength, similar to the mechanisms responsible for biochemical processes like protein folding. ${ }^{38}$ The qualitative thermodynamic model we propose here explains the role of solvents in AuNW superstructure formation and the packing density of the assemblies. It is a first step towards a detailed understanding of the ternary system of metal colloids, their ligand shells, and the dispersant. Our results suggest that the assembly of ultrathin nanowires can be exquisitely controlled through the ligand-solvent interplay. This will facilitate the assembly of AuNW building blocks into functional materials for photonic and electronic applications.

\section{Acknowledgements}

The project was funded by the German Federal Ministry of Education and Research in the "NanoMatFutur" program. Prof. Eduard Arzt is acknowledged for his continuing support.

\section{Notes and references}

1 L. Cademartiri and G. A. Ozin, Adv. Mater., 2009, 21, 1013-1020.

2 H. Feng, Y. Yang, Y. You, G. Li, J. Guo, T. Yu, Z. Shen, T. Wu and B. Xing, Chem. Commun., 2009, 1984-1986.

3 A. Kisner, M. Heggen, D. Mayer, U. Simon, A. Offenhäusser and Y. Mourzina, Nanoscale, 2014, 6, 5146-5155.
4 J. H. M. Maurer, L. González-García, B. Reiser, I. Kanelidis and T. Kraus, Nano Lett., 2016, 16, 2921-2925.

5 S. Gong, Y. Zhao, L. W. Yap, Q. Shi, Y. Wang, J. A. P. B. Bay, D. T. H. Lai, H. Uddin and W. Cheng, Adv. Electron. Mater., 2016, 2, 1600121.

6 A. Roy, T. Pandey, N. Ravishankar and A. K. Singh, AIP Adv., 2013, 3, 032131.

7 L. Wang, P. Liu, P. Guan, M. Yang, J. Sun, Y. Cheng, A. Hirata, Z. Zhang, E. Ma, M. Chen and X. Han, Nat. Commun., 2013, 4, 2413.

8 J. Xu, H. Wang, C. Liu, Y. Yang, T. Chen, Y. Wang, F. Wang, X. Liu, B. Xing and H. Chen, J. Am. Chem. Soc., 2010, 132, 11920-11922.

9 L. Cademartiri and K. J. M. Bishop, Nat. Mater., 2015, 14, 2-9. 10 G. A. Ozin, K. Hou, B. V. Lotsch, L. Cademartiri, D. P. Puzzo, F. Scotognella, A. Ghadimi and J. Thomson, Mater. Today, 2009, 12, 12-23.

11 M. C. P. Wang and B. D. Gates, Mater. Today, 2009, 12, 34-43. 12 K. J. M. Bishop, C. E. Wilmer, S. Soh and B. A. Grzybowski, Small, 2009, 5, 1600-1630.

13 U. Landman and W. D. Luedtke, Faraday Discuss., 2004, 125, 1-22.

14 C. A. Silvera Batista, R. G. Larson and N. A. Kotov, Science, 2015, 350, 1242477.

15 C. Fasting, C. A. Schalley, M. Weber, O. Seitz, S. Hecht, B. Koksch, J. Dernedde, C. Graf, E.-W. Knapp and R. Haag, Angew. Chem., Int. Ed., 2012, 51, 10472-10498.

16 D. A. Uhlenheuer, K. Petkau and L. Brunsveld, Chem. Soc. Rev., 2010, 39, 2817-2826.

17 B. Yuan and L. Cademartiri, J. Mater. Sci. Technol., 2015, 31, 607-615.

18 S. Hajiw, J. Schmitt, M. Impéror-Clerc and B. Pansu, Soft Matter, 2015, 11, 3920-3926.

19 R. Klajn, K. J. M. Bishop and B. A. Grzybowski, Proc. Natl. Acad. Sci. U. S. A., 2007, 104, 10305-10309.

20 N. R. Jana, L. Gearheart and C. J. Murphy, Adv. Mater., 2001, 13, 1389-1393.

21 N. Goubet, J. Richardi, P.-A. Albouy and M.-P. Pileni, Adv. Funct. Mater., 2011, 21, 2693-2704.

22 L. Cademartiri, K. J. M. Bishop, P. W. Snyder and G. A. Ozin, Philos. Trans. R. Soc., A, 2012, 370, 2824-2847.

23 A. Loubat, M. Impéror-Clerc, B. Pansu, F. Meneau, B. Raquet, G. Viau and L.-M. Lacroix, Langmuir, 2014, 30, 4005-4012.

24 S. Mourdikoudis and L. M. Liz-Marzán, Chem. Mater., 2013, 25, 1465-1476.

25 J. Borges, J. A. Ribeiro, E. M. Pereira, C. A. Carreira, C. M. Pereira and F. Silva, J. Colloid Interface Sci., 2011, 358, 626-634.

26 F. London, Trans. Faraday Soc., 1937, 8-26.

27 S. A. Simon and T. J. McIntosh, Biochim. Biophys. Acta, Biomembr., 1984, 773, 169-172.

28 P. Moutet, L.-M. Lacroix, A. Robert, M. Impéror-Clerc, G. Viau and L. Ressier, Langmuir, 2015, 31, 4106-4112.

29 G. Tresset, PMC Biophys., 2009, 2, 3.

30 P. A. Korevaar, C. Schaefer, T. F. A. de Greef and E. W. Meijer, J. Am. Chem. Soc., 2012, 134, 13482-13491. 
31 H.-J. Jeon, C. Kim and H. H. Song, Macromol. Res., 2012, 20, 954-959.

32 B.-H. Wu, H.-Y. Yang, H.-Q. Huang, G.-X. Chen and N.-F. Zheng, Chin. Chem. Lett., 2013, 24, 457-462.

33 K. W. Herwig, B. Matthies and H. Taub, Phys. Rev. Lett., 1995, 75, 3154-3157.

34 C. A. Hunter and J. K. M. Sanders, J. Am. Chem. Soc., 1990, 112, 5525-5534.
35 J. A. Berrocal, F. Di Meo, M. García-Iglesias, R. P. J. Gosens, E. W. Meijer, M. Linares and A. R. A. Palmans, Chem. Commun., 2016, 52, 10870-10873.

36 Y. Yu, F. Cui, J. Sun and P. Yang, Nano Lett., 2016, 16, 3078-3084.

37 C. Wang, Y. Hu, C. M. Lieber and S. Sun, J. Am. Chem. Soc., 2008, 130, 8902-8903.

38 K. A. Dill, Biochemistry, 1990, 29, 7133-7155. 